\title{
Derechos reproductivos y libertad de las mujeres. Observaciones sobre el debate feminista*
}

\section{Isabel Fanlo Cortés**}

Resumen: Este artículo busca contribuir a la reflexión sobre el tema de los derechos reproductivos, asumiendo como perspectiva de análisis el debate feminista occidental contemporáneo. No obstante la variedad de enfoques y posiciones que caracterizan ese debate, se enfatizará el interés convergente de la discusión bioética feminista más reciente en el tema de la regulación jurídica que los Estados prevén, tanto en relación con el uso de determinadas tecnologías reproductivas como respecto del ejercicio de otros derechos reproductivos, al igual que las premisas de valor que sostienen estas políticas del derecho y sus efectos en la vida de las mujeres. Finalmente, también a la luz de las transformaciones recientes de las modalidades de control público sobre las elecciones reproductivas, se individualizarán algunos retos que siguen interrogando a los feminismos contemporáneos.

\footnotetext{
Precedentes versiones de este escrito fueron presentadas el 28 de febrero de 2014 en el ámbito del Máster sobre Gobernanza y Derechos Humanos en la Universidad Autónoma de Madrid y el ro de septiembre de 2014 en el ámbito del ciclo de Seminarios de Teoría y Filosofía del Derecho "Luis Villar Borda" en la Universidad Externado de Colombia. Agradezco a todas y todos aquellos que participaron en tales iniciativas por las críticas y sugerencias. Un agradecimiento especial para Silvana Álvarez, Emilssen González de Cancino, Diego Moreno Cruz y Pablo Moreno Cruz. Fecha de recepción: io de abril de 2017. Fecha de aceptación: 22 de mayo de 20 i 7. Para citar el artículo: Fanlo Contés, I., "Derechos reproductivos y libertad de las mujeres. Observaciones sobre el debate feminista", Revista de Derecho Privado, Universidad Externado de Colombia, n. ${ }^{\circ} 32$, enero-junio de 201 7, 29-52. DOI: https://doi.org/Io. I860 I/or $234366 . n 32.02$

* Doctorado en Derechos Humanos. Profesora asociada de Sociología del Derecho, Università degli Studi di Genova, Génova, Italia. Contacto: isa.fanlo@unige.it
} 
Palabras clave: derechos reproductivos, teorías feministas, aborto, maternidad subrogada, tecnologías reproductivas.

\section{Reproductive Rights and Women's Freedom. Remarks on the Feminist Debate}

Aвstract: This article seeks to contribute to the issue of reproductive rights, assuming for the analysis the perspective of the contemporary Western feminist debate. Notwithstanding the variety of approaches and positions that find expression in the scope of such a debate, emphasis is given to the convergent interest of the most recent feminist bioethical discussion in the subject of legal regulation that the States predict, both in relation to the use of certain Reproductive technologies, and the exercise of other reproductive rights, as well as the value premises that underpin these policies and their effects on the lives of women. Finally, in the light of the recent transformations of the modalities of public control over reproductive choices, some challenges will be identified that fall on contemporary feminisms.

Keywords: Reproductive rights, feminist theories, abortion, surrogacy, reproductive technologies.

Sumario: Introducción. I. Elecciones reproductivas entre viejas cuestiones y nuevas técnicas. II. Regulación de las técnicas reproductivas y transformación de las modalidades de control. III. Algunos (relativamente) nuevos retos para los feminismos contemporáneos. A. Recuperación del feminismo como práctica política. B. Herramientas para descifrar la complejidad. Bibliografía.

\section{Introducción}

En este trabajo planteo algunas reflexiones sobre el tema de los derechos reproductivos, es decir, el conjunto de derechos que involucran las elecciones procreativas de los individuos ${ }^{\mathrm{I}}$. Asumiré como perspectiva de análisis el debate feminista

El núcleo central de tal conjunto de derechos consiste básicamente en un derecho de libertad: la libertad de decidir si tener hijos o, en cambio, no tenerlos. Alrededor de tal derecho (relativo al si reproducirse) -que puede ser ulteriormente especificado, p. ej., en el derecho a decidir "el número de hijos, el espaciamiento de los nacimientos y el intervalo entre estos", tal como lo dispone el literal e del inciso i. ${ }^{\circ}$ del artículo i 6 de la Convención sobre la Eliminación de Todas las Formas de Discriminación contra la Mujer, aprobada por la Asamblea General de las Naciones Unidas en I979- gira una serie de derechos prestacionales que son instrumentales al libre ejercicio de las elecciones reproductivas. Entre estos derechos, a los que corresponderían deberes prestacionales a cargo de las instituciones, sobresalen el derecho a la salud reproductiva y el derecho al acceso a toda la información relativa a los métodos contraceptivos y a las técnicas disponibles de procreación médicamente asistida. En el nivel de las fuentes normativas (nacionales y supranacionales), las referencias a la expresión "derechos reproductivos" son muy raras: entre estas se acostumbra citar dos instrumentos de soft law aprobados por las Naciones 
(occidental) que ha dado, y sigue dando, contribuciones importantes a la discusión sobre este tema. Se trata de un debate muy articulado del que, obviamente, podré ocuparme solo en algunos de sus aspectos.

Las problemáticas de la procreación siempre han sido objeto de gran interés para las teorías feministas. De hecho, se trata de problemáticas que, como trataré de resaltar en la parte final de mi escrito, siguen "interrogando" al feminismo contemporáneo, generando, a su vez, desde mi punto de vista, (relativamente) nuevos e interesantes retos.

\section{Elecciones reproductivas entre viejas cuestiones y nuevas técnicas}

La razón del interés de los movimientos feministas en las elecciones reproductivas se puede intuir fácilmente. En efecto, aunque están en juego elecciones que obviamente no involucran solo a las mujeres (sino a todos los individuos, independientemente de su género y orientación sexual), es un hecho que, hasta ahora, la reproducción de seres humanos tiene lugar, principalmente, o al menos en mayor y diferente medida, en el cuerpo femenino ${ }^{2}$. Lo anterior permite a las mujeres el ejercicio de un control, un poder sobre la reproducción: un poder que, por cierto, históricamente se ha tratado de limitar mediante un esfuerzo por disciplinar los cuerpos femeninos de forma mucho más intensa que en lo referido al cuerpo masculino. Básicamente, como el pensamiento feminista ha resaltado, de ese poder femenino (i.e., poder generativo) dependen muchos de los dispositivos patriarcales de control sobre la sexualidad y, en general, la vida de las mujeres.

También en este ámbito de discusión, el feminismo no es expresión de una posición unívoca: la esfera de las elecciones reproductivas que involucran a las mujeres es y, sobre todo, ha sido terreno de inquietudes coincidentes, pero también terreno de un enfrentamiento entre orientaciones a menudo muy lejanas.

Este enfrentamiento, esta divergencia de posiciones tal vez ha sido menos encendida en relación con un tema que ha ocupado mucho la reflexión feminista: es decir, el tema de la interrupción voluntaria del embarazo. En efecto, el movi-

Unidas, donde, por primera vez en una declaración oficial, se habla de "derechos reproductivos" en términos de derechos humanos. Me refiero al Programa de Acción de la Conferencia Internacional sobre la Población y el Desarrollo (celebrada en El Cairo del 5 al I 3 de septiembre de I994), cap. viI, par. 7-3, y a la Plataforma de Acción de la Cuarta Conferencia Mundial sobre la Mujer (celebrada en Beijing del 4 al $5_{5}$ de septiembre de I995), par. 96. Por cierto, el concepto de "derechos reproductivos" empleado en estos documentos programáticos se refiere solo a aquellos derechos relativos a si reproducirse (llamados "derechos reproductivos negativos", como el derecho de acceso a los contraceptivos), dejando a un lado las elecciones relativas a cómo reproducirse y, en particular, al acceso a las técnicas de procreación médicamente asistidas.

2 Como bien me hizo notar Emilssen González de Cancino, la cuestión está destinada a asumir una relevancia diferente en el caso, aún por venir, del útero artificial. 
miento feminista se ha mostrado muy compacto al condenar la penalización de las prácticas abortivas, consciente también de los graves riesgos para la salud de la mujer como consecuencia del aborto clandestino ${ }^{3}$.

$\mathrm{Al}$ menos en atención al panorama italiano, si bien algunas feministas estaban no solo a favor de la despenalización sino también a favor del aborto libre, mientras muchas otras no eran favorables a esta última opción, la disciplina actualmente vigente sobre la materia ${ }^{4}$ las desilusionó a todas, puesto que el legislador limitó la despenalización del aborto solo a aquellos casos en que, en el sentir del médico, exista un riesgo para la salud física y/o síquica de la mujer ("serio" en los primeros 90 días de embarazo y "grave" después), tomando en escasa consideración su derecho a la autodeterminación5.

Del mismo modo, y más allá de las soluciones institucionales, también en el plano de la discusión filosófica y moral existe una cierta convergencia por parte del pensamiento feminista más reciente en el sentido de alejarse críticamente del modo tradicional como se enfoca el problema del aborto, es decir, en términos de conflicto entre derechos en cabeza de sujetos (percebidos como) "separados" o "separables": los derechos (a la autodeterminación, a la intimidad personal, a la privacy, etc.) de la madre versus los derechos (a la vida) del embrión

3 Bergallo, P., "La liberalización del aborto: contextos, modelos regulatorios y argumentos para su debate”, en Id. (comp.), Aborto y justicia reproductiva, Buenos Aires, Del Puerto, 20 I I.

4 La referencia es a la Ley I 94 aprobada por el Parlamento italiano en el año I978. Dicha ley no prevé una liberalización del aborto, ni su despenalización, sino que se limita, más bien, a establecer las condiciones en las cuales el mismo no debe ser considerado un delito punible en los términos del código penal. Para profundizar en el plano técnico-jurídico tal disciplina ver ZaNснетti, M., L'interruzione volontaria della gravidanza. Commentario sistematico alla legge 22 maggio I978 n. I94, Cedam, Padova, I992 y Borsellino, P., Bioetica tra "morali" e diritto, Raffaello Cortina, Milano, 2009, cap. 8. La disciplina italiana sobre el tema de la interrupción voluntaria del embarazo es fruto de un animado debate político y puede ser considerada como el resultado de un compromiso entre diferentes instancias, entre las cuales prevalecieron aquellas favorables a la tutela de la salud de la mujer, adoptada por el Partido Comunista Italiano. De cualquier modo, se trata de un compromiso poco representativo de la reflexión feminista de aquellos años y de la reflexión sucesiva. Sobre tales reflexiones en Italia ver, al menos, las contribuciones de Рітсн, T., Un diritto per due, il Saggiatore, Milano, ı998; Boccia, M. L., La differenza politica. Donne e cittadinanza, il Saggiatore, Milano, 2002; D'Elia, C., L'aborto e la responsabilità, Ediesse, Roma, 2008.

5 Esto, por cierto, encuentra una expresa manifestación en los principios prescritos por el artículo I de la Ley I94/i 978 donde se dispuso que el Estado no solo "garantiza el derecho a la procreación consciente y responsable" y "reconoce el valor social de la maternidad", sino que también "tutela la vida humana desde su inicio". Aunque es verdad que, como precisa Corrado del Bò, por lo menos en contextos en que la relación médico-paciente no es conflictual, y la voluntad abortiva es fuerte, los vínculos impuestos por la ley a los fines de la licitud del aborto pueden resultar, en los hechos, poco exigentes (DeL Bò, C., "L'interruzione volontaria della gravidanza", en Poggi, F. (ed.), Diritto e bioetica. Questioni fondamentali, Carocci, Roma, 20 I 3, I I I- I 27 , espec. I I 8), también es cierto que, en relación con la concreta posibilidad para las mujeres de servirse de los servicios garantizados por la misma ley, el amplio recurso del personal sanitario italiano a la objeción de conciencia (casi el 90\% en algunas regiones italianas), prevista por el artículo 9 de la Ley i94, tiene un peso muy relevante (al respecto ver Saporiti M., La coscienza disubbidiente. Ragioni, tutele e limiti dell'obiezione di coscienza, Giuffrè, Milano, 20 I4). 
o del feto ${ }^{6}$. En lugar de una visión conflictualista, algunas feministas prefieren adoptar una postura relacional que, aunque seguramente no niega que la última palabra sobre la elección de interrumpir o continuar un embarazo corresponda a la mujer, conduce a conceptualizar el derecho de elección de la mujer de manera diferente respecto a la postura liberal estándar ${ }^{7}$. En efecto, este derecho de elección es entendido, no tanto como un derecho de libertad "negativa" (decidir sobre su propio cuerpo sin interferencia ajena), sino como un derecho a tomar una decisión moral en relación con la continuación de una particular (e inescindible) relación con la/el nascitura/us, con la consciencia de que el propio cuerpo es también un lugar de vínculos y relaciones (no solo con el feto) ${ }^{8}$. Según una ética contextualista (que, entonces, toma en consideración el contexto concreto en el que la decisión debe ser tomada), el feto no desaparece en cuanto portador de intereses confluyentes respecto a la madre, sino que cobra relevancia como parte de una relación en la que la mujer es el único sujeto en condiciones de asumir decisiones responsables. Bajo esta óptica, la moralidad del aborto es defendida mediante el reconocimiento, en cabeza de la mujer, como detentadora del poder de dar vida, de una específica competencia moral: aquella de elegir si continuar o interrumpir una relación, asumiendo las consecuencias de sus propios actos. Obviamente, el concepto de relación relevante en el ámbito de este enfoque filosófico-moral no se reduce al mero concepto biológico de relación entre cuerpo materno y embrión que se origina automáticamente desde el momento de la concepción, sino que involucra una dimensión emotiva y moral, cuya protagonista e intérprete solo puede ser la mujer en estado de embarazo:

6 En realidad, las primeras autoras feministas que se ocuparon del tema se alejaron de este enfoque tradicional: ver, p. ej., las conocidas contribuciones de Thomson, J. J., "A Defense of Abortion" (r97 I) y Warren, M. A., "On Moral and Legal Status of Abortion” (i 973), ambas publicadas en Feinberg, J. (ed.), The Problem of Abortion, Wandsworth, Wandsworth Publishing, i984.

7 Esta postura debe mucho a la redefinición del concepto de autonomía personal en términos de autonomía 'relacional', ya hace tiempo elaborado en el ámbito de la reflexión feminista: ver Baier, A., Postures of the Mind. Essays on Mind and Morals, University of Minnesota Press, Minneapolis, I 985 y, más recientemente, los escritos compilados en Mackenzie, C. y Stoljar, N. (eds.), Relation Autonomy. Feminist Perspectives on Autonomy, Agency, and the Social Self, Oxford University Press, New York-Oxford, 2000. No es fácil formular una definición unívoca de 'autonomía relacional', en cuanto tal locución cumple el papel de umbrella term, bajo la cual caen todas las versiones de autonomía que comparten el hecho de asumir que el individuo ejerce las propias elecciones en el contexto de relaciones interpersonales y dependiendo de procesos de socialización que influencian su actuar. Sobre el tema ver también el trabajo de Álvarez MediNA, S., "El umbral de autonomía. La concepción relacional y la construcción de las opciones", en Hierro, L. L. (ed.), Autonomía individual frente a autonomía colectiva. Derechos en conflicto, Marcial Pons, Madrid, 20 4, 53-79.

8 Con esta perspectiva ver, entre las otras, Mackenzie, C., "Abortion and embodiment", Australian Fournal of Philosophy, 70, I992, I36-I 55; Sherwin, S., No Longer Patient: Feminist Ethics and Health Care, Temple University Press, Philadelphia, I992; Gibson, S., "The problem of abortion: Essentially contested concepts and moral autonomy", Bioethics, XVII, 3, 2004, 22 I-2 33; Bотті, C., Prospettive femministe. Morale, bioetica e vita quotidiana, Espress, Torino, 2012 , espec. I09-I I 7 . 
el único sujeto capaz de evaluar, en el caso concreto, la eventual insostenibilidad de continuar con el embarazo y con la experiencia de la maternidad, así como de asumir la responsabilidad de tal decisión?.

$\mathrm{Si}$, como decía, en relación con el tema del aborto, tanto en el plano de la discusión jurídica como en el de la discusión moral, las divergencias entre las distintas posturas feministas nunca han sido muy fuertes, en cambio, al menos hasta finales de los años noventa, fue mucho más profunda la ruptura al interior de los movimientos feministas como consecuencia de la introducción de los métodos de control de la fertilidad y de las nuevas tecnologías reproductivas.

Con esta expresión ('tecnologías reproductivas') se hace referencia a una variedad de intervenciones que van desde los contraceptivos hasta la diagnosis prenatal; desde la fecundación in vitro hasta la maternidad subrogada. Técnicas muy diferentes que suscitan problemas morales, jurídicos, sociales de diferente complejidad, acomunados, sin embargo, por un elemento común: la intervención humana sobre el proceso reproductivo, un proceso que escapa, entonces, por primera vez, a la naturaleza.

El reto al orden natural amplía, no solo el campo de nuestra libertad sino, y en consecuencia, dado que las elecciones reproductivas involucran también a otros sujetos, el ámbito de nuestra responsabilidad. Los progresos biomédicos cuestionan nuestras convicciones más profundas, suscitando reacciones, a menudo ambivalentes, que oscilan entre la esperanza y el miedo: la esperanza de lograr la liberación de un destino no deseado, pero que es impuesto por los límites naturales, y el miedo a que estos progresos puedan generar dinámicas y consecuencias que escapan peligrosamente de nuestro control ${ }^{\text {Io }}$.

En el pensamiento feminista el problema ha sido advertido con particular intensidad. En efecto, se han formulado diferentes preguntas: ¿la reproducción tecnológica representa un medio de liberación o un instrumento de opresión para las mujeres? ¿Se traduce, en otros términos, en una más amplia libertad de elección o constituye una ulterior forma de control? Si analizamos las respuestas maduradas durante los últimos treinta años nos encontramos, como ya indiqué, con enfoques muy diferentes.

9 No obstante la centralidad que en la óptica feminista asume el punto de vista de las mujeres (competentes "para decidir, para sí y, entonces, para todos, sobre el ámbito de la reproducción": Piтch, Un diritto per due, cit., 83, trad. propia), esa postura relacional no oculta el conflicto que puede suscitarse entre madre y padre en el ámbito de la decisión abortiva (a diferencia de lo que sucede en algunas discusiones en las que el problema del aborto parece consistir solo en defender los intereses del feto frente al capricho femenino). Como bien precisa Вотті (Prospettive etiche femministe, cit., espec. I I4-I I 5), reconocer la interacción (eventualmente conflictual) entre madre y padre, y también su respectiva posición asimétrica respecto a la decisión de abortar (y, entonces, las razones por las que la última palabra sobre la reproducción corresponde a las mujeres), no quiere decir que se niegue la palabra a los varones; por el contrario, al reconocerse la asimetría y las respectivas competencias, se puede definir el espacio en que la palabra de los hombres puede darse.

Battaglia, L., Bioetica senza dogmi, Rubettino, Soveria Mannelli, 2009. 
Las primeras feministas que en Europa y, sobre todo, en Estados Unidos se ocuparon del argumento (me refiero a la década de I970) saludaron con entusiasmo estas novedades como instrumentos importantes para la liberación de las mujeres y la ampliación de su autonomía procreativa. De hecho, (aunque solo en algunos casos minoritarios) con un optimismo, por decir lo menos, exagerado: en este sentido el famoso texto de Shulamith Fireston, Dialectic of Sex, publicado en r97 I, que influenció ampliamente la ciencia ficción feminista, difundió la idea según la cual únicamente el liberarse de la reproducción y su delegación a las nuevas tecnologías podría conducir a la total liberación femenina ${ }^{\mathrm{II}}$.

En cambio, el feminismo de la segunda generación, o de la diferencia, en el curso de los años i980, acusando de ingenuidad a las feministas de la primera generación, enfatizó los riesgos que suponían las nuevas tecnologías reproductivas, en términos de un aumento de control sobre los cuerpos y las elecciones de las mujeres. En algunos casos esta actitud anti-tecnológica se expresó de manera muy extrema, como, por ejemplo, en el caso de Gene Corea, quien interpreta estas novedades como medios empleados por el patriarcado para expropiar a las mujeres de su capacidad generativa y reducir el cuerpo materno a objeto o materia $^{\mathrm{I} 2}$.

Este contraste es, por cierto, la señal de divergencias teóricas más profundas, referidas al diverso modo de interpretar las diferencias de género.

El feminismo de la primera ola (o feminismo de la igualdad o de la emancipación) considera las diferencias de género, sobre todo, como diferencias socialmente construidas y como sinónimo de discriminación. En este marco, el feminismo de la primera ola está, entonces, muy atento a reivindicar la función materna como una elección, y no como un destino impuesto e inevitable; perspectiva, esta última, que ha servido para relegar a las mujeres a la esfera privada, excluyéndolas de la vida política y de las esferas decisorias.

En cambio, el feminismo de la diferencia, que empieza su desarrollo en los años I980, a menudo transformando las diferencias entre géneros en motivo de orgullo, tiende a celebrar, a exaltar el rol materno como una característica no solo exclusiva, sino también constitutiva de la identidad femenina. Esto, al menos, en algunas de sus versiones esencialistas ${ }^{13}$. Bajo esta óptica, la maternidad se configuraría como una experiencia originaria, un valor primario de reivindicación contra la cultura patriarcal: como tal, una experiencia que no admite

i I Firestone, S., Dialectic of Sex, The Case for Feminist Revolution, Morrow Quill Paperbacks, New York, I970.

I2 CoReA, G., The Mother Machine. Reproductive Technologies from Artificial Insemination to Artificial Wombs, Harper \& Row, New York, 1979.

I3 Chrodow, N., The Reproduction of Mothering. Psycoanalysis and Sociology of Gender, University of California Press, Berkeley, i978; Ruddick, S., "Maternal thinking", Feminist Studies, 6, I980, 342-367. Sobre el tema ver también Ripoli, M., "Madre natura. Considerazioni sulla maternità", Materiali per una storia della cultura giuridica, xxIv, I, I994, 23 I-248. 
injerencias por parte del poder médico y de la ciencia; poderes que son vistos, por el contrario, con profunda sospecha, en cuanto empresa masculina activada por una ideología de dominación y de control de la naturaleza y del cuerpo femenino.

\section{Regulación de las técnicas reproductivas y transformación de las modalidades de control}

Ahora bien, tomando en cuenta las variadas expresiones del feminismo contemporáneo referidas a los derechos reproductivos, me parece claro que estas resultan lejanas, tanto del optimismo tecnológico que había marcado el pensamiento de, al menos, algunas feministas de la igualdad, como de la actitud anti-tecnológica que caracterizaba buena parte del feminismo de la diferencia ${ }^{\mathrm{I}}$.

Sobre este último aspecto parece claro, por ejemplo, el decisivo distanciamiento por parte del feminismo contemporáneo frente a la demonización de la tecnología, en particular en materia reproductiva.

En efecto, el feminismo contemporáneo considera que dicha demonización, como afirma Anne Donchin, "impide prestar atención al esfuerzo de comprender y, entonces, de cambiar las condiciones materiales y las relaciones que dominan las investigaciones y los tratamientos de la infertilidad"15. Además, y con razón, según mi parecer, la mayoría de las feministas contemporáneas miran con profundo escepticismo el fundamento de la utilidad del sacrificio requerido a las mujeres estériles de renunciar al uso de tecnologías reproductivas en función de supuestos intereses colectivos (i.e., las mujeres como grupo social) ${ }^{\mathrm{I}}{ }^{\mathrm{6}}$.

En términos más amplios, se registra un distanciamiento respecto al determinismo tecnológico que era un aspecto común tanto al optimismo tecnológico de las primeras feministas como al pesimismo tecnológico del feminismo de la segunda ola. Con esta expresión -“determinismo tecnológico"- me refiero a la creencia en el absoluto condicionamiento de la tecnología sobre la vida humana. Una creencia, por cierto, que tenía como resultado común el "banalizar el rol y

I4 En este sentido también Casalini, B., "Libere di scegliere? Patriarcato, libertà e autonomia in una prospettiva di genere", Etica \& Politica, XIII, 2, 20 I I, 329-364, espec. 338-339.

I 5 Donchin, A., "Potere e procreazione: prospettive femministe nei confronti delle nuove tecnologie riproduttive", en Bioetica. Rivista interdisciplinare, 3, I996, 40 I-424, espec. 4IO.

i6 Como sugerían, por ejemplo, las exponentes del FINRRAGE (Femminist International Network of Resistence to Reproductive and Genetic Engineering) -promotoras de estudios empíricos y muy activas políticamente entre los años I980 y I990-, la renuncia a las tecnologías reproductivas, aunque constituye un sacrificio para algunas mujeres, era el precio que había que pagar para contrastar la apropiación de la función reproductiva por parte del establishment médico y su objetivo de alcanzar sus propios intereses económicos y profesionales. Ver, entre otras, Rowland, R., Living Laboratories: Women and Reproductive Technologies, Indiana University Press, Bloomington, I 992; Spallone, P., Beyond Conception: The New Politics of Reproduction, Macmillan, London, i 989 y el volumen colectivo de Arditti, R., Duelli Klein, R. y Minden, S. (eds.), Test-tube Women: What Future for Motherhood?, Pandora Press, Boston, I 984. 
el poder decisorio de la mujer vista singularmente, negando, en los hechos, en nombre de un supuesto sujeto colectivo, su capacidad de escoger y de actuar autónomamente con base en intereses que podrían, también, disentir de las estrategias globales de los diferentes feminismos" ${ }^{17}$.

Me parece que este distanciamiento por parte del feminismo contemporáneo puede ser explicado a partir de un cambio de perspectiva importante. En concreto, el "problema" es identificado, no en las técnicas reproductivas en sí mismas sino, más bien, en su gobierno y en las relaciones de poder que entran en juego en la práctica de estas técnicas y los conflictos que de allí puedan derivar.

Con esto no quiero afirmar que al interior del pensamiento feminista no haya posiciones muy críticas con referencia, por ejemplo, a los efectos de la medicalización del cuerpo femenino que pueden descender del recurso a las nuevas técnicas reproductivas. Nuevas técnicas que, en ocasiones, se limitan a actualizar prácticas sociales ya existentes desde hace tiempo: es el caso, por cierto muy controvertido al interior del debate feminista, de la maternidad subrogada, hoy ampliamente difundido a escala global. Si algunas autoras, fieles a la orientación liberal, ven en tal práctica una herramienta de empowerment de las mujeres en cuanto capaz de ampliar sus oportunidades de elección de planes de vida, muchas otras han resaltado la matriz patriarcal que puede ser identificada en la exigencia, sobre todo referida al género masculino, de reproducirse para transmitir/ perpetuar el propio patrimonio genético ${ }^{18}$ (exigencia que, según sugieren estudios estadísticos, en algunos casos conduciría a preferir el recurso a la gestación subrogada y no a la práctica de la adopción ${ }^{19}$ ). Esto, además de las numerosas críticas en relación con las pretensiones económicas de los acuerdos comerciales de gestación ${ }^{20}$. En efecto, este tipo de acuerdos - y sobre el punto volveré más adelante-, basándose a menudo en la disparidad de punto de partida de las partes del contrato (los que pretenden la subrogación y la mujer contratada), pueden convertirse en vehículos de explotación de las mujeres más débiles.

i Battaglia, L., "Il punto di vista delle donne nelle pratiche di procreazione artificiale", en Romano, C., Grassani, G. (eds.), Bioetica, Utet, Torino, I995, 345-35 I, espec. 348.

I8 En efecto, valdría la pena preguntarse, siguiendo a Katz Rothman, si en una sociedad matrilineal la práctica de la gestación por subrogación despertaría el mismo interés; ver KaTz Rothman, B., "The legacy of patriarchy as context for surrogacy. Or why are we quibbling over this?", The American fournal of Bioethics , xIV, 5, 2014, 36-37, disponible en: https://drive.google. com/file/d/oB 5 cvFHon3yAudtvbu IJocHdyrta/view

I9 Sobre este aspecto ver Pozzolo, S., "Locatioventris", Bioderecho, 3, 20I6, 37-64, espec. par. v.

20 Por ejemplo, algunas autoras equiparan los acuerdos comerciales de gestación con formas de esclavitud o con contratos de venta de niños. Sobre el punto ver las referencias en ANDrEws, L. B., "Surrogate motherhood: The challenge for feminists", Law Medicine \& Health Care, I6, I988, 72-8o. Recientemente, algunas voces feministas muy críticas frente a la maternidad subrogada (también en la forma "altruista") se han expresado, por ejemplo, en el ámbito de un debate muy vivaz en España (http://nosomosvasijas.eu/). Tal debate ha enfrentado a estos movimientos feministas con grupos y asociaciones LGBTI que, en cambio, reivindican la legalización de la práctica, hoy prohibida en España por la Ley de Reproducción Asistida del año 2006. 
No obstante las críticas mencionadas, la preocupación más difundida no parece ser la de condenar moralmente dicha práctica ${ }^{2}$, sinoque, más bien, se concentra en el modo en que, a nivel institucional, la maternidad subrogada es regulada (o no regulada) o como debería serlo ${ }^{22}$ : esto también con base en la razonable idea según la cual, dado que tal práctica existe desde hace siglos, la misma debe ser necesariamente disciplinada a nivel nacional y supranacional, teniendo en consideración los diferentes intereses en juego (del niño o de la niña, de los padres de intención y, obviamente, los intereses, muchas veces desatendidos, de la madre gestante) $)^{23}$.

2 I Obviamente, con las debidas excepciones. Como se indicaba también en la nota precedente, hay voces muy críticas frente a la legitimidad moral de tal práctica que es interpretada como una ulterior forma de disciplinamiento que re-propone la maternidad como un destino necesario para las mujeres (a cualquier costo) e implica un estigma social para aquellas que eligen no reproducirse o no pueden hacerlo. Para una presentación de estas tesis ver, p. ej., LindEMANN Nelson, H., "Dethroning choices: Analogy, personhood and the new reproductive technologies", Journal of Law, Medicine \& Ethics, XxiII, I995, I29-I35; Donchin, A., "Prospettive che convergono: le critiche femministe alla riproduzione assistita”, en Faralli, C. y Cortesi, C. (eds.), Nuove maternità. Riflessioni bioetiche al femminile, Diabasis, Reggio Emilia, 2005, 69-ıo8. Como es obvio, la condena de tal práctica en términos morales no necesariamente implica una posición contraria a su regulación en el plano jurídico y la admisión de ciertas formas de maternidad subrogada (como aquella voluntariamente altruista) más respetuosas, según algunas autoras, de la subjetividad de las mujeres y del valor de su experiencia gestacional (en este sentido ver, p. ej., Danna, D., Contract Children. Questioning Surrogacy, Ibidem, Stuttgart, 20 I 5).

22 Para una atenta presentación de las modalidades con que los diferentes Estados miembros de la Unión Europea han afrontado el fenómeno de la gestación subrogada, ver los reports elaborados anualmente por el Grupo de trabajo constituido en el año 20 Io al interior de The Hague Conference: https://www.hcch.net/en/projects/legislative-projects/parentage-surrogacy

$23 \mathrm{Si}$, como ya se recordó, algunas autoras abogan por limitar la licitud de la práctica solo a los casos en que la misma sea "altruista" y, entonces, cuando, a excepción de los gastos necesarios para el embarazo y el parto, el contrato con la madre gestante sea a título gratuito, otras autoras precisan que, aunque se enmarque la subrogación en el ámbito de contratos a título gratuito, en un contexto de economía capitalista existe el riesgo de una explotación de las mujeres involucradas (exceptuando los pocos casos de subrogación practicada por parientes y amigos) o, en el mejor de los casos, el riesgo de la simulación de contratos a título oneroso que, sin embargo, no prevén una tutela para la parte más débil (situación en la que, de nuevo, quienes resultarían perjudicadas serían las mujeres: de este aspecto ya se había ocupado Andrews, L. B., "Alternative modes of reproduction", en Cohen, S. y TAub, N. (eds.), Reproductive Laws of the I99os, Humana Press, Clifton, I989, 380-388). En el ámbito de la subrogación "comercial" (es decir onerosa), uno de los aspectos más cruciales, desde una perspectiva feminista, tiene que ver con la regulación jurídica del caso en que la madre gestante, después de haber estipulado el contrato con los padres intencionales, cambia de parecer y expresa la voluntad de interrumpir el embarazo, o la intención de no entregar, después del parto, al niño o a la niña que ha llevado en su vientre. En efecto, es evidente que afrontar estos casos desde el punto de vista tradicional del incumplimiento contractual es discutible desde una perspectiva feminista, aunque se trate de una solución prevista en algunos ordenamientos jurídicos donde la maternidad por subrogación es lícita. Para una introducción a las principales soluciones jurídicas que, ya desde los años I990, han sido propuestas desde una perspectiva feminista (solo en parte adoptadas en algunos ordenamientos jurídicos) ver Tong, R., "Feminist perspectives and gestational motherhood: The need for a unified legal focus", en Callahan, J. (ed.), Reproduction, Ethics, and the Law: Feminist Perspectives, Indiana University Press, Bloomington, I995, 55-79. 
En términos más generales, y como consecuencia del cambio de perspectiva al que hice referencia, en la discusión adquiere relevancia el tipo de regulación jurídica que los Estados prevén, sea respecto del uso de determinadas tecnologías reproductivas, sea respecto del ejercicio de otros derechos reproductivos, así como las premisas de valor que sostienen estas políticas del derecho y sus resultados en la vida de las mujeres.

En cuanto respecta, por ejemplo, a la procreación médicamente asistida, en países como Italia, donde está prevista una disciplina muy restrictiva (Ley 40/2004) que, por un lado, prohíbe el recurso a las tecnologías reproductivas a las personas solteras y a las parejas homosexuales y, por el otro, admitía, antes de una pronunciamiento muy reciente de Corte Constitucional ${ }^{24}$, solo procedimientos de fecundación llamada homóloga (es decir, sin recurrir a donadores externos), se ha generado un debate interesante sobre el fundamento ideológico y los efectos sociales de estos límites.

Muchas feministas han resaltado que estos límites pueden ser leídos como la expresión de la voluntad legislativa de re-legitimar, también en este ámbito, una concepción tradicional de la familia "natural", ya superada en los hechos 25 : una voluntad legislativa que, al prohibir la fecundación heteróloga y la maternidad subrogada, no solo limita fuertemente el posible éxito del recurso a las técnicas de procreación medicamente asistida, sino que somete a las mujeres a tratamientos extenuantes y, a menudo, dañosos para su salud. Además, estas prohibiciones, impuestas en nombre de un supuesto respeto de lo "natural", son discriminatorias en un doble sentido, en cuanto empujan, a quien puede, a escoger el camino del turismo reproductivo reservado a pocas, y alimentan, al mismo tiempo, un verdadero mercado a nivel global, donde, a menudo, quienes sufren las peores consecuencias son las mujeres socialmente más desfavorecidas.

El ya citado caso de las madres subrogadas, por ejemplo, sería emblemático de aquel fenómeno al que se le ha llamado la "nueva división internacional del trabajo reproductivo" 26 : de una parte, hay personas solteras o parejas provenientes de Europa, con medios económicos a disposición que, como consecuencia de

24 Me refiero a la sentencia de la Corte Constitucional italiana n. ${ }^{\circ}$ i62/2014 que declaró la inconstitucionalidad de la prohibición de la fecundación heteróloga. La Ley 40/2004, elaborada con el claro propósito de proteger al embrión, fue objeto de una incisiva reescritura por parte de la Corte Constitucional que, entre otras cosas, recientemente juzgó como "irrazonable" la prohibición del diagnóstico preimplantacional, tomando en consideración que el ordenamiento jurídico admite la legitimidad del diagnóstico prenatal y, con base en el mismo, permite la interrupción voluntaria del embarazo en caso de graves malformaciones del feto (ver sentencia n. ${ }^{\circ}$ 96/2OI5).

25 Saraceno, C., "Ma che razza di famiglia è?", il Mulino, 2, 2004, 239-244. Para una lectura igualmente crítica ver Rodotà, S., "Prefazione", en Valentini, C., La fecondazione proibita, Feltrinelli, Milano, 2004, 7-I8.

26 Yeats, N., Globalizing Care Economies and Migrant Workers. Explorations in Global Care Chains, Palgrave, Macmillan, New York, 2009, I9-20. 
normativas prohibicionistas vigentes en su país, están obligadas a viajar para lograr cumplir su (legítimo) deseo de tener un hijo. De otro lado, están las madres subrogadas, a menudo puestas a disposición por clínicas contra la infertilidad en India, Sudáfrica, Ucrania, a bajo costo (basta pensar que el costo de una madre subrogada en India gira alrededor de los Io mil dólares, contra los 50-80 mil necesarios en Estados Unidos). Y estas madres subrogadas, muchas veces viviendo en el contexto de culturas ligadas a valores tradicionales, son socialmente estigmatizadas en razón de su oficio, percibido como un trabajo "sucio", asimilable a la prostitución.

La lógica prohibicionista que, como ya dije, inspira la regulación jurídica respecto al uso de las tecnologías reproductivas en muchos países de Europa, así como los continuos esfuerzos, a veces exitosos, de retroceso respecto a las conquistas de la legalización del aborto ${ }^{27}$, son claros signos del interés del Estado por ejercer un control, cada vez más invasivo, sobre las elecciones reproductivas de las mujeres y sobre el cuerpo femenino, con el fin de determinar el espacio de lo lícito y de lo prohibido en el ámbito de la parentalidad, de la familia y de la sexualidad (por cierto, obviamente, a la misma lógica responden las prácticas de esterilización forzada que aún se practican en muchas partes del mundo).

La reflexión feminista, especialmente aquella referida al análisis de las mutaciones del neoliberalismo bajo una perspectiva de género ${ }^{28}$, ha contribuido a esclarecer cómo las modalidades por medio de las cuales se ejerce este control han cambiado como consecuencia de, entre otros, dos fenómenos estrechamente ligados entre sí.

El primer fenómeno se refiere a la afirmación de aquello que Nikolas Rose definió “ciudadanía biológica" ${ }^{29}$, es decir, la idea según la cual nuestra misma vida biológica -estado de salud o enfermedad- es objeto de elecciones, decisiones y, entonces, de responsabilidades individuales. En consecuencia, también como resultado de la crisis del Estado social, en el horizonte neoliberal, el con-

27 El ataque al derecho al aborto (bajo algunas condiciones y/o dentro de algunos plazos), derecho previsto y regulado en diferentes ordenamientos jurídicos, es un fenómeno que ha asumido, en estos últimos tiempos, una dimensión "transnacional", en cuanto involucra realidades muy distantes entre sí, no solo dentro de Europa (desde Polonia hasta Italia, pasando por Francia), sino también a nivel mundial (paradigmático el caso de Estados Unidos).

28 A este respecto ver, en el debate anglosajón, los trabajos de N. Fraser ("Feminism, Capitalism and the Cunning of History", New Left Review, 56, 2009, 97-I I 7 y Fortunes of Feminism. From State-managed Capitalism to Neoliberal Crisis, Verso, London-New York, 20 I 3), Angela McRoввIE (entre ellos The Aftermath of Feminism. Gender, Culture and Social Change, Sage, Los AngelesLondon-New Delhi-Singapore-Washington, 2009), Janet Newman (entre ellos "Spaces of power: Feminism, Neoliberalism and gendered labor", Social Politics, xx, 2, 20 I 3, 200-22 I). En Italia ver recientemente Casalini, B., "Neoliberismo e femminismi", Fura Gentium, xII, I, 20 I 5 , 3 I-65 y Dini, T. y Tarantino, S. (eds.), Femminismo e neoliberalismo. Libertà femminile versus imprenditoria di sé e precarietà, Natan Edizioni, $20 \mathrm{I} 4$.

29 Rose, N., The Politics of Life Itself: Biomedicine, Power, and Subjectivity in the Twenty-First Century, PUP, 2007. 
trol sobre la procreación de la vida biológica (objeto de un control "público" cada vez más invasivo) se da mediante un tránsito de la responsabilidad de la colectividad hacia el individuo ${ }^{\circ}{ }^{\circ}$.

Así, aunque hoy existen muchas más opciones y más información sobre la reproducción en general -desde el embarazo hasta el parto-, esta proliferación de conocimiento (que no es obviamente el objeto de la crítica) se ha traducido, no solo en una ampliación de la libertad de las mujeres, sino también en una ampliación de deberes a su cargo. En toda una serie de decisiones, que van desde el uso de la píldora anticonceptiva hasta la elección de practicarse exámenes prenatales (como la amniocentesis), o la decisión de lactar, o no, al propio hijo ${ }^{3 \mathrm{I}}$, la mujer, en cuanto madre, asume ella misma, a cargo suyo, la gestión individual del riesgo reproductivo y genético, con todas las responsabilidades que de ello derivan ${ }^{32}$.

El segundo fenómeno que ha conducido a una modificación de las modalidades de control se refiere a la difusión de un constante llamado, en el discurso público, a una libertad reproductiva y de elección que, en los hechos, también en el así llamado primer mundo, es un lujo de una minoría de mujeres. De esto último es cómplice también la crisis económica y la contracción del gasto social de estos últimos años que, como sabemos, ha acentuado la precarización del mercado del trabajo, golpeando, sobre todo, a los grupos jóvenes de la pobla-

30 Este fenómeno está íntimamente conectado con una redefinición del papel del Estado respecto del imperativo del crecimiento económico dominante en la cultura liberista: papel que se concentra en la enfatización de la idea, ya capturada por Foucault, del llamado emprendimiento de sí mismo (Foucault, M., Naissance de la biopolitique. Cours au College de France, 1978-1979, Paris, 2004). La idea, promovida por tal cultura, consistente en que los mismos individuos son conducidos a percibirse como individuos "empresarios" de sí mismos e impulsados a invertir/ potenciar su proprio capital humano (en parte constituido por elementos innatos y en parte por elementos adquiridos) según un cálculo de costos-beneficios, de modo que el eventual fracaso de tal inversión (que se refiere también a la salud del propio cuerpo y del propio patrimonio genético) es entendido como consecuencia exclusiva de elecciones individuales inapropiadas o de la incapacidad de asumir riesgos y no, en cambio, de obstáculos de tipo social. Este modelo empresarial, que sería común, también, a la retórica del llamado investment state (la "tercera vía" que se consolida con Blair y Clinton durante la segunda mitad de los años I990), se extendería también a las mujeres y, en particular, a la figura materna. Como precisa Brunella Casalini, la figura materna "se convierte en una particular figura de empresaria, en cuanto resulta cargada de la responsabilidad de gestionar y volver productivo, según una rigurosa lógica de costosbeneficios, aquel capital humano en formación que es el menor" (CASALINI, "Neoliberismo e femminismi", cit., 50-5 I, trad. propia).

3 I Sobre la cuestión de la lactancia materna ver el artículo de Forti, S. y Guaraldo, O., "Rinforzare la specie. Il corpo femminile tra biopolitica e religione materna", Filosofia politica, I, 2006, $57-78$.

32 Casalini, B., "Libere di scegliere? Patriarcato, libertà e autonomia in una prospettiva di genere", Etica \& Politica, xIII, 2, 20 I I, 329-364, espec. 337. Se trata de responsabilidades que son compartidas también con la ciencia médica: sobre el punto me permito reenviar a Fanlo CoRTÉs, I., "Giudici, responsabilità medica e controllo sulle scelte riproduttive", en D. CARUsi (ed.), Chiamati al mondo. Vite nascenti ed autodeterminazione procreativa, Torino, Giappichelli, 20I4, 97-107. 
ción: la mayoría de las mujeres en edad fértil no tienen la libertad de elegir. Por el contrario, en muchos casos, o bien están obligadas a reenviar la elección de la maternidad a los cuarenta años (precisamente cuando la curva de fertilidad desciende notoriamente), o bien lo están a renunciar a la propia realización profesional, dada la ausencia de políticas serias dirigidas a facilitar la conciliación entre los tiempos de vida y los tiempos de trabajo. En este sentido se expresan claramente los datos recogidos en Gender Equality Index Report -recientísima investigación de la Unión Europea sobre el estado de la igualdad entre hombres y mujeres- que, aunque con diferente incidencia en las diversas realidades nacionales, muestran cómo la elección de convertirse en madres ha tenido el efecto de reducir ulteriormente la presencia (ya muy limitada) de la mujer en el mercado del trabajo externo/retribuido, o, al menos, el efecto de imponerles soluciones de flexibilidad (part-time, mini jobs, etc.), caracterizadas por una deficiente protección social 33 .

\section{Algunos (relativamente) nuevos retos para los feminismos contemporáneos}

Frente a tales transformaciones, que el mismo pensamiento feminista ha contribuido a identificar, diversos son los retos, solo relativamente novedosos ${ }^{34}$, para los feminismos contemporáneos. En particular, me detendré en dos aspectos que me parecen cruciales.

\section{A. Recuperación del feminismo como práctica política}

Dejando a un lado, por el momento, el debate específico sobre los derechos reproductivos, me parece que los feminismos que en los últimos veinte años han dominado buena parte de la escena internacional del feminismo académico (con algunas importantes excepciones ${ }^{35}$ ) han alimentado una fuerte separación entre

33 Sobre estos temas, con particular referencia a la condición de las mujeres en Europa, me permito reenviar al editorial de Fanlo Cortés, I. y Pozzolo, S., "We want sex (equality). Labour market reforms, economic crisis and the condition of women in Europe", About Gender. Rivista internazionale di studi di genere, II, 4, 2013 , I-XXIV y a los escritos publicados en la sección monográfica del número de la revista apenas citada.

34 Los retos relativos a la conveniencia de recuperar la dimensión política del movimiento feminista y de privilegiar un enfoque interseccional no pueden considerarse del todo "nuevos", aunque seguramente, a la luz de su relevancia en el plano de la política del derecho y de la dimensión transnacional de los fenómenos apenas analizados, ambos están destinados, hoy más que nunca, a condicionar la agenda feminista contemporánea.

35 En Europa se ha desarrollado una reflexión feminista atenta a no aislar las propuestas teóricas y a prestar atención a sus implicaciones políticas: se trata de propuestas que han sido agrupadas, junto con ejemplos de otras latitudes (piénsese en Nancy Fraser en Estados Unidos), en la corriente del llamado por la estudiosa española Celia Amorós "feminismo neo-ilustrado". Para el caso español ver Morondo Taramund, D., "Un caffè da Starbucks. Intersezionalità e disgre- 
el plano teórico y el plano práctico, conduciendo a una segura "despolitización" del movimiento de las mujeres.

Me refiero, en particular, a la influencia de las variadas corrientes que se circunscriben en el feminismo llamado posmoderno: un fenómeno internamente heterogéneo y externamente, como todas las expresiones de lo posmoderno, con confines poco definidos ${ }^{36}$. Dicho de forma simple, este tipo de feminismo está caracterizado por un enfoque de profunda crítica y escepticismo frente a la moderna idea de sujeto y a todos los conceptos que, en la tradición filosófica occidental, sirvieron para caracterizarla: i.e., la autonomía, la responsabilidad, la intencionalidad, etc.37. En otros términos -seguramente reductivos frente a las refinadas teorías de autoras como Judith Butler o Gayatri Spivak-, la tesis epistemológica que sostiene al feminismo posmoderno es aquella de la disolución, de la desagregación del sujeto y de todas sus formas de identidad, incluida aquella del género, que constituiría una mera ficción, tras la cual se esconderían performances (actuaciones) y no rostros humanos. Según Butler el sujeto etiquetado por el género (gendered self) no existe; el género, como otras formas de identidad, no es sino "una máscara sin actor" 38 .

Pues bien, si la empresa deconstructiva de Butler, así como las de otras exponentes del feminismo posmoderno, seguramente contiene elementos teóricos de gran interés, el problema es que dichas empresas no ofrecen indicaciones (constructivas) sobre cuál debe ser el camino a seguir para una re-conceptualización de la subjetividad política de las mujeres que les permita incidir en la realidad social que las circunda. Una realidad social que, aunque para las mujeres más afortunadas es testimonio de conquistas importantes respecto al pasado, muestra claramente cómo muchos objetivos del proyecto emancipador del feminismo aún están bien lejos de ser alcanzados ${ }^{39}$. En este sentido, son un testimonio claro

gazione del soggetto nella sfida al diritto antidiscriminatorio", Ragion pratica, 37, 20 I I, 365-384, espec. 374-376 y también Morondo Taramundi, D., "Una sonda en el post-patriarcado: el debate sobre emancipación y libertà femminile en el feminismo italo-español", Gênero e Direito. Periódico do Núcleo de Estudos e Pesquisas sobre Gênero e Direito, 2, 201 5, disponible en: http:// periodicos.ufpb.br/ojs2/index.php/ged/index [consultado el I 5 de diciembre de 2016].

36 Este vínculo entre el éxito de las teorías feministas posmodernas y la despolitización del movimiento de las mujeres lo enfatiza de manera muy clara Morondo Taramundi, "Un caffè da Starbucks. Intersezionalità e disgregazione del soggetto nella sfida al diritto antidiscriminatorio", cit., 37 I-373.

37 Para una panorámica general sobre los feminismos posmodernos ver Nicholson, L. (ed.), $F e-$ minism/Postmodernism, New York-London, Routledge, I989; Flax, J., Thinking Fragments: Psychoanalysis, Feminism and Postmodernism in the Contemporary West, Berkeley, University of California Press, I990.

$3^{8}$ Butler, J., Gender Trouble: Feminism and the Subversion of Identity, New York-London, Routledge, r990.

39 Para una crítica al feminismo posmoderno ver Benhabib, S., Situating the Self. Gender, Community and Postmodernism in Contemporary Ethics, New York, Routledge, I992, espec. 203-24I; Amorós, C., Tiempo de feminismo. Sobre feminismo, proyecto ilustrado y posmodernidad, Madrid, 
los graves retrocesos que caracterizan las políticas públicas en el ámbito reproductivo (que ya mencioné), junto con otras señales preocupantes en términos de retroceso cultural.

Frente a estos retrocesos que tienden a (re)conducir a las mujeres al ámbito de lo "privado" o a entregar sus cuerpos a decisiones ajenas, creo que el feminismo debe enfrentar las cuestiones políticas y, entonces, el papel de teoría crítica que sostiene un movimiento de lucha de emancipación y liberación de mecanismos opresores. Es evidente que la emancipación, respecto de una situación de subordinación, especialmente para sujetos como las mujeres, que han sido histórica y constantemente hetero-dominadas, implica un proceso de des-identificación respecto a la diferencia genérica que les fue asignada como una construcción social y cultural. Pero esta discusión, esta capacidad crítica de distanciamiento respecto a los roles estereotipados del discurso hegemónico (a menudo reformulados también por parte de ciertos feminismos) presupone, como nos recuerda bien la estudiosa española Celia Amorós, que los seres humanos tenemos un margen de maniobra para transformar los significados constituidos, para interpretar las situaciones dadas y reconstruirlas asignándoles un sentido nuevo ${ }^{4}$. Un margen de maniobra al que, en cambio, la disolución del sujeto anunciado por el feminismo posmoderno no parece dejar ningún espacio.

Recuperar la atención del feminismo en las cuestiones políticas no significa, obviamente, descuidar el plano de la elaboración teórica.

\section{B. Herramientas para descifrar la complejidad}

Por el contrario -y me ocupo ahora del segundo aspecto- los cambios que se han producido en el ámbito reproductivo, de los modelos familiares, del mercado del trabajo y de la economía requieren, para ser descifrados, afinar los instrumentos teóricos. Estos cambios, en efecto, hacen más difícil la comprensión de la realidad, enriqueciéndola con ambigüedades y contradicciones que pueden generar equívocos e ingenuos optimismos a la hora de analizar las condiciones de las mujeres. Pienso, por ejemplo, en la tesis defendida por algunas feministas italianas según la cual la mayor feminización del espacio público y la aclamada crisis de la masculinidad serían claros síntomas de la "muerte" del patriarcado ${ }^{4}$.

Cátedra, 2000; Álvarez Medina, S., "Diferencia y teoría feminista”, en Beltrán, E. y Maquiei-

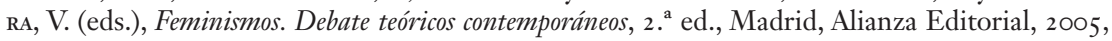
243-286, espec. $267-268$.

40 Amorós, Tiempo de feminismo, cit., 20 ss. y Morondo Taramundi, "Un caffè da Starbucks. Intersezionalità e disgregazione del soggetto nella sfida al diritto antidiscriminatorio", cit., 376.

4I Adopta esta perspectiva la corriente del "Pensiero della differenza sessuale" ("Pensamiento de la diferencia sexual") que ha tenido amplia difusión en el contexto cultural italiano y está representado por los escritos del grupo Libreria delle Donne di Milano y de Luisa Muraro. Véase, p. ej., el conocido documento producido por el grupo Libreria delle Donne di Milano en enero de i996 
$\mathrm{O}$, también, pienso en el énfasis que recientemente una parte del feminismo (el llamado choice feminism) ha hecho sobre la mayor libertad de elección de la que gozarían las mujeres respecto al pasado; una mayor libertad de elección que equivaldría a una emancipación de la condición de opresión que, hasta ahora, ha caracterizado la condición femenina. Con base en esta perspectiva, "las mujeres pueden hacer casi cualquier cosa, abandonar la carrera por la familia y los hijos, practicarse cirugías estéticas, recurrir a las nuevas tecnologías reproductivas, y seguir considerándose feministas, siempre y cuando su decisión sea el fruto de una 'elección' personal" ${ }^{2}$. Se trata de la idea, típicamente liberal, según la cual lo que cuenta es la autonomía personal, aquí entendida como la libertad del individuo de escoger sin sufrir interferencias que no sean consensualmente aceptadas, prescindiendo del contenido de las elecciones. Una idea atractiva, capaz de liberar al choice feminism de la acusación de moralismo que varias veces ha golpeado al feminismo de la segunda ola ${ }^{43}$.

$\mathrm{Y}$, sin embargo, la respuesta del choice feminism parece demasiado apresurada y, sobre todo, inadecuada para descifrar las complejidades inducidas por las profundas transformaciones ya citadas.

En primer lugar, la presencia del consenso individual, como manifestación de la autonomía personal, no elimina necesariamente el problema de la opresión, al menos no en los términos en que este problema (que no puede ser reducido al de la ausencia de coacción individual) ha sido abordado al interior del análisis feminista sobre las relaciones de poder en el sistema patriarcal y sobre el análisis de las prácticas y las estructuras sociales que de dicho sistema son expresión. Como nos recordó Iris Marion Young, la "opresión de las mujeres" no se refiere a las mujeres individualmente consideradas, sino que involucra una cuestión colectiva, i.e., la condición de discriminación de un grupo social44. Es, además, una cuestión estructural, que no depende de "las elecciones o políticas de unas pocas personas. Sus causas están ínsitas en normas, hábitos y símbolos que no se cuestionan, en los presupuestos que subyacen a las reglas institucionales y en las consecuencias colectivas de seguir esas reglas" 45 . En otras palabras, la libre elección individual no cierra la discusión, sino, más bien, deja el espacio para otros interrogantes: ¿por qué una mujer sin hijos corre el riesgo de tener que afrontar

(disponible en: http://www.libreriadelledonne.it/_oldsite/news/articoli/sottosopra96.htm) que comienza con la siguiente afirmación: "El patriarcado ha terminado, no cuenta ya con el crédito femenino y está acabado" (trad. propia).

Casalini, "Libere di scegliere? Patriarcato, libertà e autonomia in una prospettiva di genere", cit., espec. 334 .

Marso, L., "Feminism's Quest for Common Desires", Perspectives on Politics, viII, I, 2010, 263269, espec. 294. trad. de Silvina Álvarez, La justicia y la política de la diferencia, Madrid, Cátedra, 2000.

45 Ibíd., 4I. 
un estigma social, así como la mujer que desea tenerlos sin renunciar a la propia realización profesional? ¿Por qué las mujeres, al someterse a dietas extenuantes o a cirugías estéticas invasivas, deciden voluntariamente someterse a códigos de deseabilidad que son heterodeterminados por una mirada externa? ¿Hasta qué punto las mujeres logran subvertir estos códigos? ¿Cuáles son los vínculos y los obstáculos que impiden que prevalezca la propia mirada sobre sí mismas? ¿Qué genera esos vínculos y esos obstáculos?

En segundo lugar, como resalta Brunella Casalini, el choice femminism, basándose en la lógica del viejo modelo del contrato entre individuos libres, iguales e independientes, corre el riesgo de descuidar las condiciones estructurales de las relaciones económicas, sociales, políticas y culturales, al interior de las cuales se realizan las elecciones. Elecciones que, en muchos casos, siguen siendo diferentes para los hombres, las mujeres y, obviamente, entre diferentes grupos de hombres y mujeres ${ }^{4}$.

A este propósito, precisamente el caso de los derechos reproductivos enfatiza e invita a reflexionar sobre el variado impacto que las tecnologías reproductivas pueden tener sobre las mujeres del Norte y del Sur del mundo, sobre las mujeres de diferentes clases sociales y de diferentes etnias, sobre el diferente significado que las mismas mujeres atribuyen a 'libertad reproductiva'.

En términos más generales, y si bien consciente del dispositivo común que preside, en todas las partes del mundo, a los diferentes mecanismos de opresión y de control del cuerpo femenino, el feminismo contemporáneo, ahora más que nunca, debe tomar en consideración la cuestión de las diferencias entre las mujeres ampliamente tematizada por el debate sobre la llamada "interseccionalidad". Este debate, retomado a finales de los años I980 por la jurista feminista Kimberlè Crenshaw47, se ocupa de los efectos derivados del hecho de que los individuos pertenecen, al mismo tiempo, a diferentes grupos sociales, por lo que una persona nunca es solo mujer o hombre, heterosexual u homosexual, blanca o negra, con o sin discapacidad, etc., sino que es portadora de múltiples identidades, y que la super-posición de varias identidades puede ser fuente de un surplus de discriminación ${ }^{4}$.

46 Casalini, "Libere di scegliere? Patriarcato, libertà e autonomia in una prospettiva di genere", cit., 329-364, espec. 334-335.

47 Crenshaw, K., "Demarginalizing the intersection of race and sex: A black feminist critique of antidiscrimination doctrine, feminist theory and antiracist politics", University of Chicago Legal Forum, I989, I39-167 e Id., "Mapping the margins: Intersectionality, identity politics, and violence against women of color", Stanford Law Review, 43, I 99 I, I 24I-I 299. Como nos recuerda Morondo Taramund, "Un caffè da Starbucks", cit., el tema de la interseccionalidad, aunque no el término para denotarlo, no era nuevo en el pensamiento feminista. Véase, p. ej., Hull, G. et al. (eds.), All the Women are White, All the Blacks are Men, But Some of Us are Brave, New York, The Feminist Press, I982.

48 Para interesantes análisis aplicativos de la perspectiva interseccional en el ámbito europeo ver, recientemente, el número monográfico dedicado a la Intersezionalità tra diritto e società, coordi- 
Bajo ese perfil, las acusaciones de etnocentrismo que, ya a partir de los años I970, el feminismo negro y, después, el poscolonial y el lésbico han dirigido contra el feminismo occidental mainstream (hablar en nombre de todas, si bien representan solo el punto de vista de las mujeres heterosexuales-blancas-económicamente privilegiadas) han marcado un importante punto de fractura, poniendo definitivamente en crisis cualquier pretensión de asumir la categoría "mujer" en términos homogéneos y totalizadores.

Estos son solo algunos de los retos que el complejo tema de los derechos reproductivos plantea de nuevo, en todo su alcance, a los feminismos contemporáneos.

\section{Bibliografía}

Álvarez Medina, S., "Diferencia y teoría feminista”, en Beltrán, E. y Maquieira V. (eds.), Feminismos. Debates teóricos contemporáneos, 2. a ed., Madrid, Alianza Editorial, 2005, 243-286.

Álvarez Medina, S., "El umbral de autonomía. La concepción relacional y la construcción de las opciones”, en Hierro, L. L. (ed.), Autonomía individual frente a autonomía colectiva. Derechos en conflicto, Madrid, Marcial Pons, 20I4, 53-79.

Aмоrós, C., Tiempo de feminismo. Sobre feminismo, proyecto ilustrado y posmodernidad, Madrid, Cátedra, 2000.

Andrews, L.B., "Surrogate motherhood: The challenge for feminists", Law Medicine \& Health Care, I6, I988, 72-80.

Andrews, L.B., "Alternative modes of reproduction", en Cohen, S. y Taub, N. (eds.), Reproductive Laws of the I99os, Clifton, Humana Press, I989, 380-388.

Arditti, R.; Duelli Klein, R. y Minden, S. (eds.), Test-tube Women: What Future for Motherhood?, Boston, Pandora Press, I984.

Baier, A., Postures of the Mind. Essays on Mind and Morals, Minneapolis, University of Minnesota Press, I985.

nado por Bello, B. G. y Mancini, L. (eds.), Sociologia del diritto, 2, 20 i6, acompañado, también, por una entrevista a Kimberlé Crenshaw. 
Battaglia, L., "Il punto di vista delle donne nelle pratiche di procreazione artificiale", en Romano, C. y Grassani, G. (eds.), Bioetica, Torino, Utet, I995, 345-35 I.

Battaglia, L., Bioetica senza dogmi, Soveria Mannelli, Rubettino, 2009.

Bello, B. G. y Mancini, L. (eds.), "Intersezionalità tra diritto e società", Sociologia del diritto, 2, 2016 (n. ${ }^{\circ}$ monográfico).

Benhabib, S., Situating the Self. Gender, Community and Postmodernism in Contemporary Ethics, New York, Routledge, I992.

Bergallo, P., "La liberalización del aborto: contextos, modelos regulatorios y argumentos para su debate", en Id. (comp.), Aborto y justicia reproductiva, Buenos Aires, Del Puerto, 20 I I.

Boccia, M. L., La differenza politica. Donne e cittadinanza, Milano, il Saggiatore, 2002.

Borsellino, P., Bioetica tra "morali" e diritto, Milano, Raffaello Cortina, 2009.

Botтi, C., Prospettive femministe. Morale, bioetica e vita quotidiana, Torino, Espress, 2012 .

Butler, J., Gender Trouble: Feminism and the Subversion of Identity, New YorkLondon, Routledge, I990.

Casalini, B., "Libere di scegliere? Patriarcato, libertà e autonomia in una prospettiva di genere", Etica \& Politica, XIII, 2, 201 I, 329-364.

Casalini, B., "Neoliberismo e femminismi”, fura Gentium, xiI, I, 201 5, 3 I-65.

Chrodow, N., The Reproduction of Mothering. Psycoanalysis and Sociology of Gender, Berkeley, University of California Press, 1978.

Corea, G., The Mother Machine: Reprductive Technologies from Artificial Inseminarion to Artificial Wombs, New York, Harper and Row, 1985.

Crenshaw, K., "Demarginalizing the intersection of race and sex: A black feminist critique of antidiscrimination doctrine, feminist theory and antiracist politics", University of Chicago Legal Forum, I989, I39-167. 
Crenshaw, K., "Mapping the margins: Intersectionality, identity politics, and violence against women of color", Stanford Law Review, 43, I 99 I, I 24 I-I 299.

Danna, D., Contract Children. Questioning Surrogacy, Stuttgart, Ibidem, 20 I 5.

D’Elia, C., L'aborto e la responsabilità, Roma, Ediesse, 2008.

Del Bò, C., "L'interruzione volontaria della gravidanza”, en Poggi, F. (ed.), Diritto e bioetica. Questioni fondamentali, Roma, Carocci, 20 I 3, I I I-I 27.

Dini, T. y Tarantino, S. (eds.), Femminismo e neoliberalismo. Libertà femminile versus imprenditoria di sé e precarietà, Natan Edizioni, 2014.

Donchin, A., "Potere e procreazione: prospettive femministe nei confronti delle nuove tecnologie riproduttive", Bioetica. Rivista interdisciplinare, 3, I996, $40 I-424$.

Donchin, A., "Prospettive che convergono: le critiche femministe alla riproduzione assistita”, en Faralli, C. y Cortesi, C. (eds.), Nuove maternità. Riflessioni bioetiche al femminile, Reggio Emilia, Diabasis, 2005, 69-ıо8.

Dworkin, R., Life's Dominion. An argument about abortion, euthanasia and individuale freedom, New York, Knopf, I993.

Fanlo Cortés, I. y Pozzolo, S., "We want sex (equality). Labour market reforms, economic crisis and the condition of women in Europe", AG. About Gender. Rivista internazionale di studi di genere, II, 4, 201 3, I-XXIV.

FAnlo Cortés, I., "Giudici, responsabilità medica e controllo sulle scelte riproduttive", en Carusi, D. (ed.), Chiamati al mondo. Vite nascenti ed autodeterminazione procreativa, Torino, Giappichelli, 2014, 97-107.

Flax, J., Thinking Fragments: Psychoanalysis, Feminism and Postmodernism in the Contemporary West, Berkeley, University of California Press, I990.

Forti, S. y Guaraldo, O., "Rinforzare la specie. Il corpo femminile tra biopolitica e religione materna", Filosofia politica, I, 2006, 57-78.

Foucault, M., Naissance de la biopolitique. Cours au College de France, I978-1979, Paris, 2004. 
Fraser, N., "Feminism, Capitalism and the Cunning of History", New Left Review, 56, 2009, 97-I I 7.

Fraser, N., Fortunes of feminism. From State-managed capitalism to neoliberal crisis, Verso, London-New York, 2013.

Gibson, S., "The problem of abortion: Essentially contested concepts and moral autonomy", Bioethics, XVII, 3, 2004, 22 I-233.

Katz Rothman, B., "The legacy of patriarchy as context for surrogacy. Or why are we quibbling over this?", The American fournal of Bioethics, xIV, 5, 2014.

Lindemann Nelson, H., "Dethroning choices: Analogy, personhood and the new reproductive technologies", Journal of Law, Medicine \& Ethics, xxIII, I995, I 29-I35.

Mackenzie, C., "Abortion and embodiment", Australian Fournal of Philosophy, 70, I 992 , I $36-$ I 55 .

Mackenzie, C. y Stoljar, N. (eds.), Relation Autonomy. Feminist Perspectives on Autonomy, Agency, and the Social Self, New York-Oxford, Oxford University Press, 2000.

MacRoвbie, A., The aftermath of feminism. Gender, culture and social change, Los Angeles-London-New Delhi-Singapore-Washington, Sage, 2009.

Marso, L., "Feminism's quest for common desires", Perspectives on Politics, viII, I, 2010, 263-269.

Morondo Taramundi, D., "Un caffè da Starbucks. Intersezionalità e disgregazione del soggetto nella sfida al diritto antidiscriminatorio", Ragion pratica, 37,201 I, $365-384$.

Morondo Taramundi, D., "Una sonda en el post-patriarcado: el debate sobre emancipación y libertà femminile en el feminismo italo-español", Gênero e Direito. Periódico do Núcleo de Estudos e Pesquisas sobre Gênero e Direito, 2, 20 I 5 , disponible en: http://periodicos.ufpb.br/ojs2/index.php/ged/index [consultado el i 5 de diciembre 20I6].

Nicholson, L. (ed.), Feminism/Postmodernism, New York-London, Routledge, I989. 
Рітсн, T., Un diritto per due, Milano, il Saggiatore, I998.

Pozzolo, S., "Locatioventris", Bioderecho, 3, 2016, 37-64.

Pozzolo, S. (ed.), "Debating 'surrogacy' or pregnancy for others. A first round of opinions", AG. About Gender. Rivista internazionale di studi di genere, v, Io, 20I6, 33 I-357.

Roberts, D., Killing the Black Body. Race, Reproduction, and the Meaning of Liberty, New York, Vintage Books, I999.

Ripoli, M., "Madre natura. Considerazioni sulla maternità", Materiali per una storia della cultura giuridica, xxIv, I, I994, 23 I-248.

Rodotà, S., "Prefazione”, en Valentini, C., La fecondazione proibita, Milano, Feltrinelli, 2004, 7-I 8.

Rowland, R., Living Laboratories: Women and Reproductive Technologies, Bloomington, Indiana University Press, I 992.

Rose, N., The Politics of Life Itself: Biomedicine, Power, and Subjectivity in the TwentyFirst Century, Pup, 2007.

Ruddick, S., "Maternal thinking”, Feminist Studies, 6, I980, 342-367.

Saraceno, C., "Ma che razza di famiglia è?", il Mulino, 2, 2004, 239-244.

Saporiti, M., La coscienza disubbidiente. Ragioni, tutele e limiti dell'obiezione di coscienza, Milano, Giuffrè, 2014.

Sherwin, S., No Longer Patient: Feminist Ethics and Health Care, Philadelphia, Temple University Press, I992.

Spallone, P., Beyond Conception: The New Politics of Reproduction, London, Macmillan, I989.

Thomson, J. J., "A defense of abortion” (i97 I), en Feinberg, J. (ed.), The Problem of Abortion, Wandsworth, Wandsworth Publishing, I984.

Tong, R., "Feminist perspectives and gestational motherhood: The need for a unified legal focus", en Callahan, J. (ed.), Reproduction, Ethics, and the Law: Feminist Perspectives, Bloomington, Indiana University Press, I 995, 55-79. 
Warren, M. A., "On moral and legal status of abortion”, en Feinberg, J. (ed.), The Problem of Abortion, Wandsworth, Wandsworth Publishing, I984.

Young, I. M., Fustice and the Politics of Difference, Princeton, N.J., Princeton University Press, I990, trad. Silvina Álvarez, La justicia y la política de la diferencia, Madrid, Cátedra, 2000.

Zanchetti, M., L'interruzione volontaria della gravidanza. Commentario sistematico alla legge 22 maggio 1978 n. I94, Padova, Cedam, I992. 